\title{
Co-expression of peripheral olfactory receptors with SARS-CoV-2 infection mediators: Potential implications beyond loss of smell as a COVID-19 symptom
}

\author{
RACHEL KERSLAKE ${ }^{1}$, MARCIA HALL ${ }^{1,2}$, HARPAL S. RANDEVA ${ }^{3-5}$, DEMETRIOS A. SPANDIDOS ${ }^{6}$, \\ KAMALJIT CHATHA $^{5,7}$, IOANNIS KYROU ${ }^{3-5^{*}}$ and EMMANOUIL KARTERIS ${ }^{1 *}$ \\ ${ }^{1}$ Biosciences, College of Health and Life Sciences, Brunel University London, Uxbridge UB8 3PH; \\ ${ }^{2}$ Mount Vernon Cancer Centre, Northwood HA6 2RN; ${ }^{3}$ Warwickshire Institute for the Study of Diabetes, \\ Endocrinology and Metabolism (WISDEM), University Hospitals Coventry and Warwickshire NHS Trust, \\ Coventry CV2 2DX; ${ }^{4}$ Aston Medical Research Institute, Aston Medical School, Aston University, \\ Birmingham B4 7ET; ${ }^{5}$ Warwick Medical School, University of Warwick, Coventry CV4 7AL, UK; \\ ${ }^{6}$ Laboratory of Clinical Virology, Medical School, University of Crete, 71409 Heraklion, Greece; \\ ${ }^{7}$ Department of Biochemistry and Immunology, University Hospitals Coventry \\ and Warwickshire NHS Trust, Coventry CV2 2DX, UK
}

Received May 29, 2020; Accepted June 17, 2020

DOI: $10.3892 /$ ijmm.2020.4646

\begin{abstract}
Severe acute respiratory syndrome (SARS) coronavirus-2 (SARS-CoV-2) enters into human host cells via mechanisms facilitated mostly by angiotensin-converting enzyme 2 (ACE2) and transmembrane protease serine 2 (TMPRSS2). New loss of smell (anosmia/hyposmia) is now recognized as a COVID-19 related symptom, which may be caused by SARS-CoV-2 infection and damage of the olfactory receptor (OR) cells in the nasal neuro-epithelium and/or central involvement of the olfactory bulb. ORs are also expressed peripherally (e.g., in tissues of the gastrointestinal and respiratory systems) and it is possible that their local functions could also be impaired by SARS-CoV-2 infection of these tissues. Using Gene Expression Profiling Interactive Analysis, The Cancer Genome Atlas, Genotype-Tissue Expression, cBioPortal and Shiny Methylation Analysis Resource Tool, we highlight the expression of peripheral ORs in both healthy and malignant tissues, and describe their co-expression with key mediators of SARS-CoV-2 infection,
\end{abstract}

Correspondence to: Dr Emmanouil Karteris, Biosciences, College of Health and Life Sciences, Brunel University London, Kingston Lane, Uxbridge UB8 3PH, UK

E-mail: emmanouil.karteris@brunel.ac.uk

*Contributed equally

Key words: olfactory receptors, pan-cancer, DNA methylation, COVID-19, angiotensin-converting enzyme 2, transmembrane protease serine 2, cathepsin $\mathrm{L}$, prostate cancer, anosmia, SARS-CoV-2 such as ACE2 and TMPRSS2, as well as cathepsin L (CTSL; another cellular protease mediating SARS-CoV-2 infection of host cells). A wide expression profile of peripheral ORs was noted, particularly in tissues such as the prostate, testis, thyroid, brain, liver, kidney and bladder, as well as tissues with known involvement in cardio-metabolic disease (e.g., the adipose tissue, pancreas and heart). Among these, OR51E2, in particular, was significantly upregulated in prostate adenocarcinoma (PRAD) and co-expressed primarily with TMPRSS2. Functional networks of this OR were further analysed using the GeneMANIA interactive tool, showing that OR51E2 interacts with a plethora of genes related to the prostate. Further in vitro and clinical studies are clearly required to elucidate the role of ORs, both at the olfactory level and the periphery, in the context of COVID-19.

\section{Introduction}

The ongoing COVID-19 pandemic caused by the severe acute respiratory syndrome (SARS) coronavirus-2 (SARS-CoV-2) infection has affected over 6.5 million people thus far, resulting in the death of over four hundred thousand individuals worldwide (1). Recently, new loss of the sense of smell, either total (anosmia) or partial (hyposmia), has been recognised as a symptom of COVID-19 by the World Health Organisation (WHO) and the US Centres for Disease Control and Prevention (CDC) (www.cdc.gov/coronavirus/2019-ncov/symptoms-testing/symptoms.html).

Accordingly, additional research focus has now been placed on exploring anosmia and the involvement of olfactory receptors (ORs) in COVID-19 (2). Although poorly defined, more than 400 functional ORs are expressed in the human body, with corresponding ligands which remain mostly unclassified (3). Thought to be located primarily in 
the olfactory epithelium of the nasal cavity, these G-Protein Coupled Receptors (GPCRs) are also known to be expressed in peripheral tissues (4). As such, in humans, ORs are involved in additional processes/pathways other than those for smell perception, which have been identified to mediate physiological functions in the cardiovascular, gastrointestinal and respiratory system $(5,6)$. For example, ORs appear to be involved in widespread chemosensory systems, with OR51E2 expressed in the airway smooth muscle acting on processes which lead to increased airway smooth muscle mass that is a hallmark of asthma (4). Of note, the severity of COVID-19 shows a positive association with certain comorbidities which affect these tissues/systems, including asthma, obesity, diabetes and cancer (7).

Fusion of the SARS-CoV-2 spike proteins with the host transmembrane receptor angiotensin-converting enzyme 2 (ACE2) is shown to instigate spike protein cleavage through interaction with cellular proteases, such as transmembrane protease serine 2 (TMPRSS2), consequently allowing viral entry into the host cell $(8,9)$. Additional intracellular proteases, such as cathepsin L (CTSL), might also mediate host cell infection by SARS-CoV-2 $(8,9)$. Upon entry, the virus is then able to cause cellular damage and instigate a response which may result in a spectrum of clinical sequelae, ranging from anosmia to pneumonia, respiratory failure, cardiovascular distress and death (10).

Given the negligible expression of ACE2 and TMPRSS2 in olfactory neuronal cells and substantial expression in the olfactory epithelium, it has been postulated that sustentacular cells (supporting cells) are also involved in local viral entry and anosmia (11). Additional evidence including the expression of ACE2 and TMPRSS2 by the olfactory mucosa supports the notion that SARS-CoV-2 is capable of infecting non-neuronal cell types and, thus, subsequently disrupt odour perception (12).

The present study aimed to identify, both in normal and cancer tissues, the co-expression profile of a number of ORs which are known to be expressed in peripheral tissues in relationship to key mediators of the SARS-CoV-2 infection, namely ACE2, TMPRSS2 and CTSL. As such, concentrating on non-neuronal (peripheral) expression of ORs, here we provide a comprehensive analysis of the expression profile of ORs in peripheral tissues, several of which co-express ACE2, TMPRSS2 and CTSL and are correspondingly associated with comorbidities predisposing to severe COVID-19 (Fig. 1).

\section{Data collection methods}

Bioinformatic analysis. Expression analysis of ACE2, CTSL, TMPRSS2, OR51E2, OR10Q1, OR2A1, OR2W3, OR1J4, OR2A7, OR1Q1, OR6A2, OR1J1 and OR4M1 were validated through the Genotype-Tissue Expression (GTEx), The Cancer Genome Atlas (TCGA) and GEPIA (http://gepia.cancer). Information regarding TCGA cohort pan-cancer data and methylation data acquired through cBioPortal (http://www. cbioportal.org/) and Shiny Methylation Analysis Resource Tool (SMART) (http://www.bioinfo-zs.com/smartapp). Network localisation data were acquired through GeneMANIA software (http://genemania.org). Data sets accessed for pan-cancer analysis: ACC, adrenocortical carcinoma; BLCA, bladder urothelial carcinoma; BRCA, breast invasive carcinoma; CESC, cervical squamous cell carcinoma and endocervical adenocarcinoma; CHOL, cholangiocarcinoma; COAD, colon adenocarcinoma; DLBC, lymphoid neoplasm diffuse large B cell lymphoma; ESCA, oesophageal carcinoma; GBM, glioblastoma multiforme; HNSC, head and neck squamous cell carcinoma; $\mathrm{KICH}$, kidney chromophobe; KIRC, kidney renal clear cell carcinoma; KIRP, kidney renal papillary cell carcinoma; LAML, acute myeloid leukaemia; LGG, brain lower grade glioma; LIHC, liver hepatocellular carcinoma; LUAD, lung adenocarcinoma; LUSC, lung squamous cell carcinoma; MESO, mesothelioma; OV, ovarian serous cystadenocarcinoma; PAAD, pancreatic adenocarcinoma; PCPG, pheochromocytoma and paraganglioma; PRAD, prostate adenocarcinoma; READ, rectum adenocarcinoma; SARC, sarcoma; SKCM, skin cutaneous melanoma; STAD, stomach adenocarcinoma; TGCT, testicular germ cell tumours; THCA, thyroid carcinoma; THYM, thymoma; UCEC, uterine corpus endometrial carcinoma; UCS, uterine carcinosarcoma and UVM, uveal melanoma.

\section{Results}

Using GTEx Multi Gene Query expression, data was obtained for the assessment of ten ORs in normal tissues (Fig. 2). These data show that: OR51E2 is primarily expressed in the prostate, arteries and colon; OR10Q1 is expressed in adipose tissue, breast, salivary gland, pancreas and testis; OR2A1 exhibited a much wider distribution in adipose tissue, arteries, breast, fibroblasts, cervix, lung, tibial nerve, ovary, skin, thyroid, uterus and vagina; OR2W3 is primarily expressed in tibial nerve, brain, thyroid and whole blood; OR1J4 is primarily expressed in the bladder, fibroblasts, cervix, lung, salivary gland, and testis; OR2A7 is highly expressed in the bladder, breast, colon, oesophagus, skin, small intestine, thyroid, lung and vagina; OR1Q1 is primarily expressed in the bladder and fibroblasts; OR6A 2 exhibited high expression in the bladder, testis, thyroid, uterus and vagina; OR1J1 is primarily expressed in the bladder, fibroblasts, and testis. Information regarding the expression of OR4M1 in healthy tissues is limited (expression in testis only) with mapping and classification being an ongoing process.

Fig. 3 presents the GTEx gene expression data regarding the co-expression of ORs with ACE2, TMPRSS2 and CTSL in multiple normal tissues, including the lungs, oesophagus, salivary gland, colon, testis, thyroid and kidney, as well as tissues with established involvement in cardio-metabolic syndrome (e.g., the heart, pancreas and adipose tissue). These data corroborate recent findings showing relatively high expression of ACE2 and CTSL in the majority of tissues assessed, with varying levels of TMPRSS2 recorded $(13,14)$.

Given that cancer is concidered as a comorbididity which predisposes to severe COVID-19, we also investigated the pan-cancer expression of ORs, including data regarding methylation and mutational profiles. Using the TCGA datasets for all cancers, only four ORs appeared to be significantly differentially regulated (Fig. 4). OR2A1 was down-regulated in OV and THCA; OR2W3 was down-regulated in THYM; OR2A7 was down-regulated in SKCM and THCA; whereas OR51E2 was down-regulated in COAD and READ but up-regulated in PRAD. 

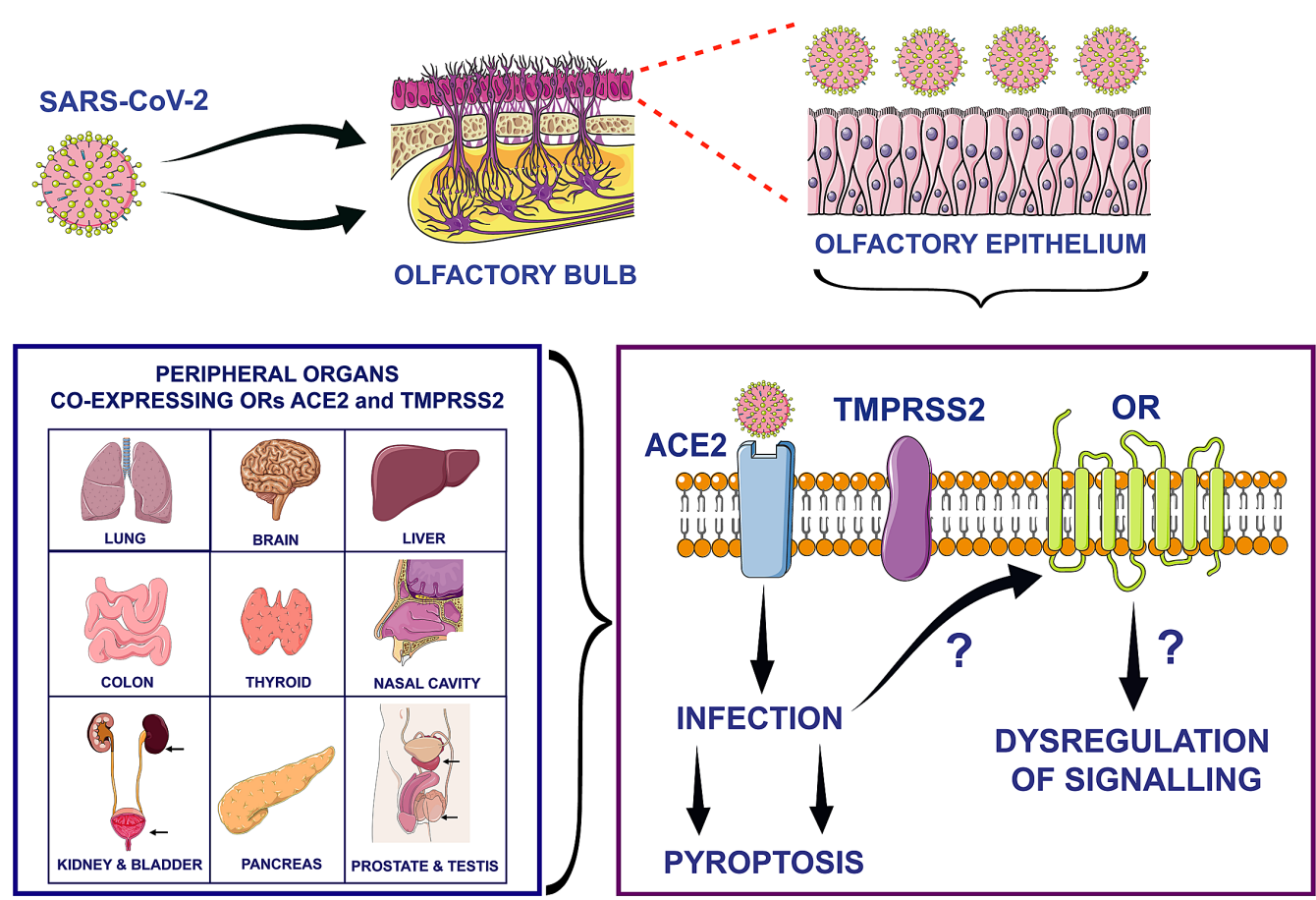

Figure 1. Following entry of SARS-CoV-2 into host cells which is facilitated by ACE2 and TMPRSS2, the function of ORs could be potentially impaired (in part or completely) in infected olfactory and peripheral tissues where ORs are also expressed. The present research highlights the expression of peripheral ORs in both healthy and malignant tissues and identifies their co-expression with key mediators of SARS-CoV-2 infection, such as ACE2 and TMPRSS2. Further research is required to explore whether this co-expression could potentially have additional COVID-19 related consequences due to damage of infected cells expressing ORs and/or impaired OR function in these peripheral tissues, in a similar way to SARS-CoV-2 infection causing loss of smell (anosmia) which is now recognized as a COVID-19 related symptom. ACE2, angiotensin-converting enzyme 2; TMPRSS2, transmembrane protease serine 2; ORs, olfactory receptors.

Subsequently, using the SMART tool, the promoter methylation status of these ORs in comparison to ACE2, TMPRSS2 and CTSL was also investigated (Fig. 5). Data sets without comparable normal tissue have been removed (all OR methylation data can be viewed in Fig. S1). Lower promoter methylation often indicates higher protein gene expression due to reduced inhibition of the promoter region. However, despite the extensive data regarding decreased methylation status within an array of data sets, when compared with GEPIA expression data, changes in methylation status of nine ORs did not significantly correlate with changes in gene expression. Of the differentially methylated genes, only OR51E2 exhibits both lower tumour methylation and significant increase in gene expression in PRAD (Fig. 4).

Finally, the data from cBioPortal revealed that the highest level of alterations in the data set for these ORs relates to gene amplification or mutation across the cancer panel (Fig. 6). Supplementary Fig. S2 presents further breakdown of this mutational profile. Of note, melanoma appears to have a high frequency of OR alterations compared to the other cancer types, while the only OR to present with an alteration in the form of a fusion is OR51E2 in PRAD.

\section{Discussion}

In this in silico study, we present novel evidence regarding the peripheral tissue distribution of ORs and their co-expression pattern in relationship to key mediators of SARS-CoV-2 infection. The potential involvement of ORs in the loss of smell as one of the COVID-19 presenting symptoms has been hypoth- esized since the first clinical cases of COVID-19 patients with anosmia (15). Based on our present findings, it is plausible that SARS-CoV-2 infection may exert damage and impair the function of ORs, not only in the nasal epithelium, but also peripherally with wider implications.

Indeed, given the known expression and involvement of the airway smooth muscle ORs in bronchodilation and airway relaxation (5); such peripheral expression of ORs may have additional implications relating to COVID-19. As such, damage and potential interference with signalling pathways associated with OR functions, as noted for nasal epithelial ORs, may contribute to underlying mechanisms predisposing to adverse COVID-19 related clinical outcomes in patients with certain comorbidities (e.g., asthma or cancer) (16). In addition, our findings suggest potential links which should be explored in the context of male preponderance for severe COVID-19, given that a number of ORs were overexpressed in testes. Indeed, the latter is an organ prone to infections by other viruses, including HIV, hepatitis or papilloma among other, which can cause viral orchitis or even lead to testicular cancer (17). Therefore, the possibility that the testes represents an additional target organ for SARS-CoV-2 merits further investigation.

The notion that SARS-CoV-2 infection might induce transcriptional changes of ORs is also supported by available data regarding effects of SARS-CoV infection on gene expression profiles. For example, a study by Reghunathan et al (18) in patients with SARS showed that this viral infection can alter the expression of immune response genes. SARS-CoV transfected monocytes have been shown to exhibit changes 

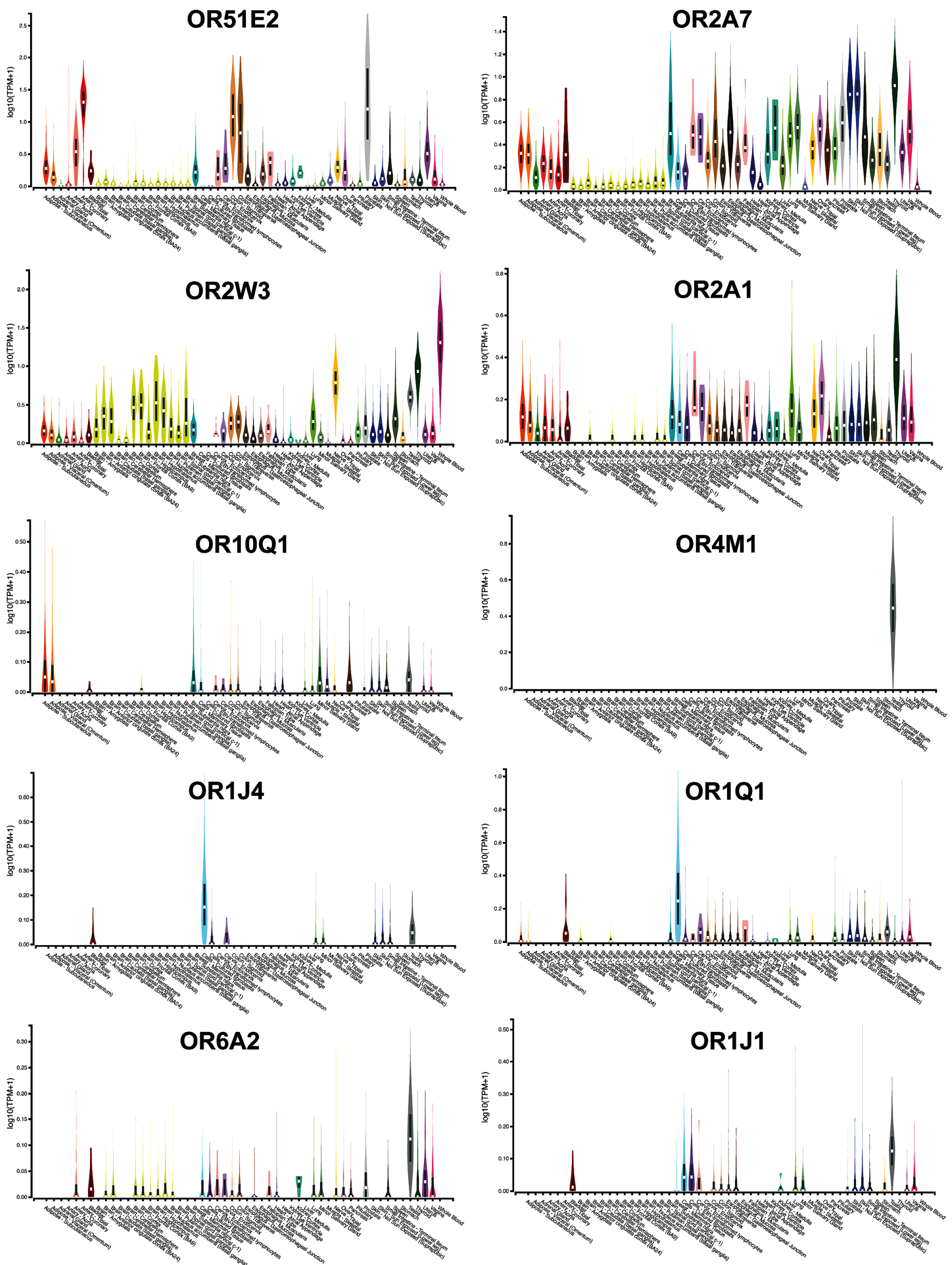

Figure 2. Expression of ORs in normal peripheral tissues using the GTEx database. Wide range of expression was noted for OR51E2, OR2A7, OR10Q1, OR1Q1, OR2A1, OR6A2 and OR2W3. OR2W3 shows the highest level of tissue specific expression, whilst OR1J1 and OR1J4 show minimal levels of protein expression. ORs, olfactory receptors. 
$\operatorname{TPM}^{0.0} \quad \begin{array}{llllll}2.4 & 10 & 37 & 1.3 \mathrm{e}+2 & 4.3 \mathrm{e}+2\end{array}$

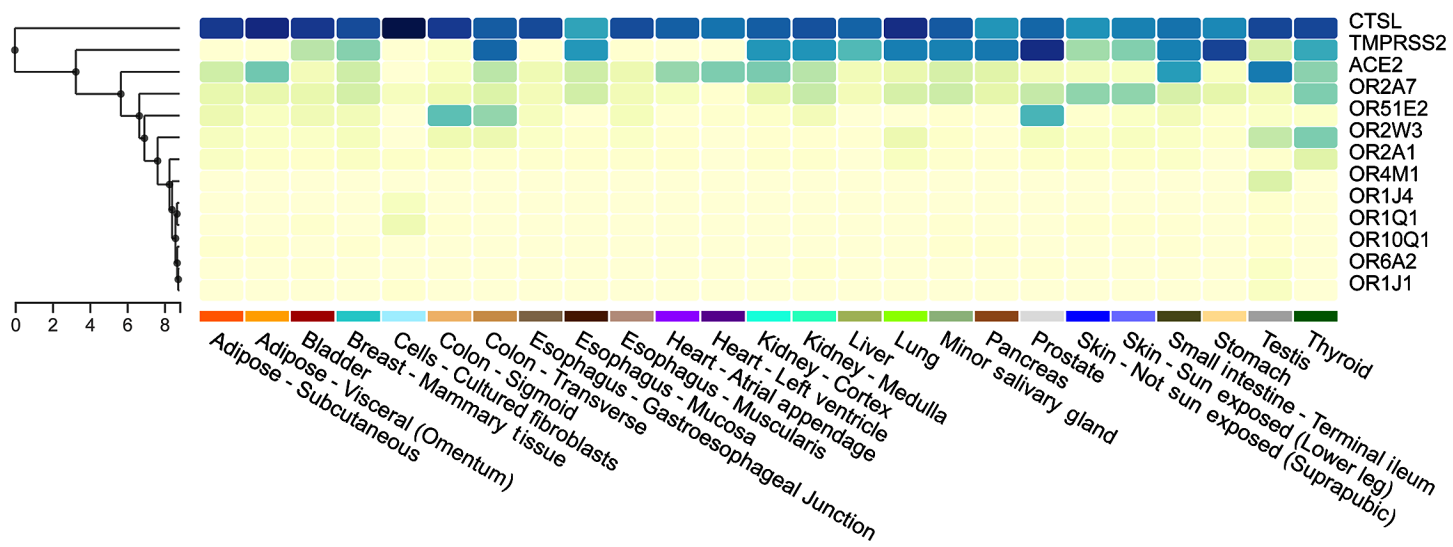

Figure 3. GTEx Multi Gene Query Map showing the co-expression of of ACE2, TMPRSS2, CTSL and peripheral ORs in normal tissues. Relitively high co-expression of ORs in relation to ACE2, TMPRSS2 and CTSL is documented in the colon, testis and thyroid. CTSL is vastly expressed throughout the full range of these tissues. ACE2, angiotensin-converting enzyme 2; TMPRSS2, transmembrane protease serine 2; CTSL, cathepsin L; ORs, olfactory receptors.
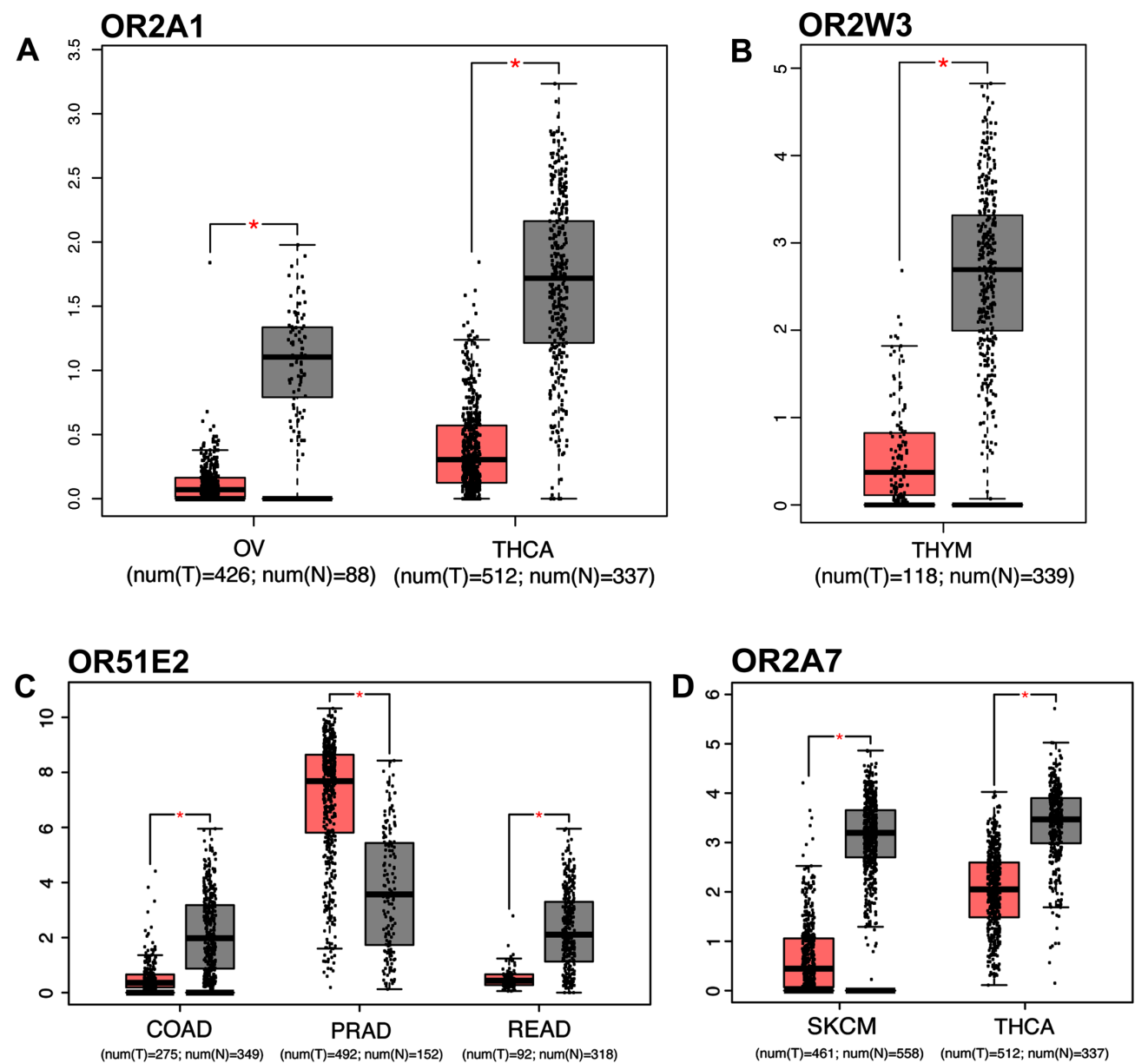

Figure 4. Pan-cancer expression of ORs. (A) Significant decrease in the expression of OR2A1 in OV and THCA tumours (Red) compared to normal (Grey). (B) Significant decrease in tumour expression of OR2W3 for THYM. (C) Significant increase in expression of OR51E2 in PRAD and decrease in COAD and READ tumour data set. (D) Significant decrease of OR2A7 in both SKCM and THCA. ORs, olfactory receptors; OV, ovarian serous cystadenocarcinoma; THCA, thyroid carcinoma; THYM, thymoma; PRAD, prostate adenocarcinoma; COAD, colon adenocarcinoma; READ, rectum adenocarcinoma; SKCM, skin cutaneous melanoma.

in immune-related genes, such as interferons, cathepsin and cytokine-related signalling genes (19). Of note, murine lung epithelial cells infected by another coronavirus (MHV-1) have been shown to exhibit certain gene expression changes, including downregulation of an OR (OLFR291), and changes in other components downstream of GPCR signalling (20).

In the present study, a comprehensive map of differential methylation of ORs across a wide repertoire of cancers is 


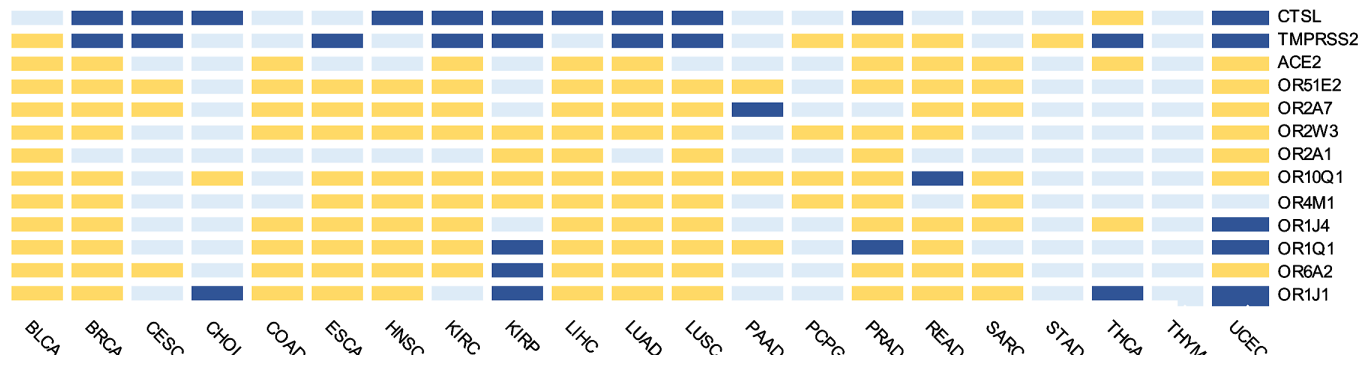

Figure 5. Pan-cancer methylation analysis of OR genes co-expressed with ACE2, TMPRSS2 and CTSL. CTSL: higher expression across a number of tumour sets (blue). ORs: the majority of data sets indicate lower methylation within tumour samples (yellow). High percentage of differentially methylated ORs across 14 cancers within the panel. No statistically significant difference detected (light blue). Data sets with an absence of control were omitted from this graph. ORs, olfactory receptors, ACE2, angiotensin-converting enzyme 2; TMPRSS2, transmembrane protease serine 2; CTSL, cathepsin L.
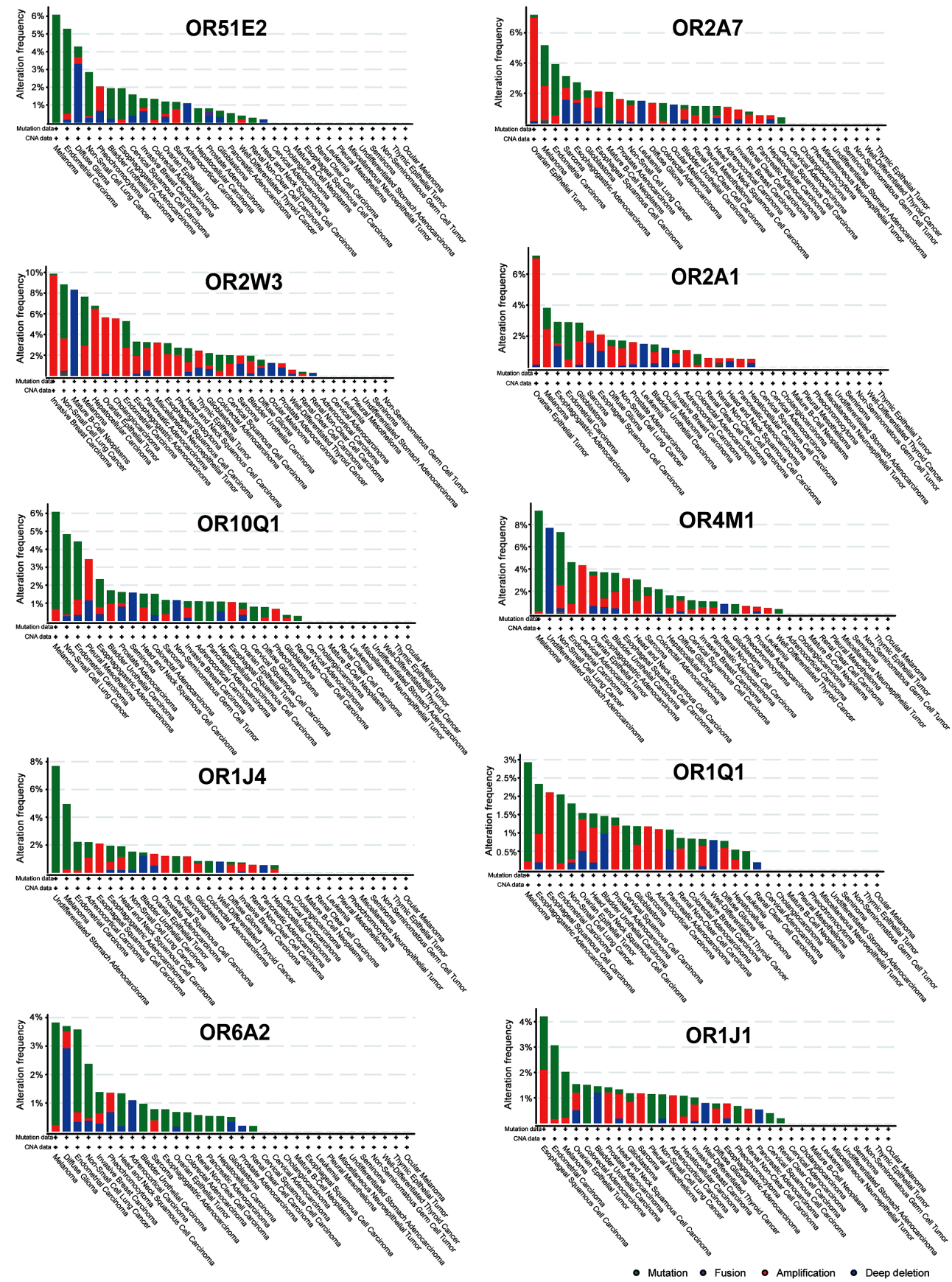

Figure 6. Pan-cancer view of OR gene alterations (cBioPortal). OR51E2, expressed a high number of mutations and a fusion in the prostate; OR2A7-OR2A1, alterations are primarily amplification; OR10Q1, range of amplifications, most frequent level of mutations recorded in melanoma and non-small cell lung cancer; OR4M1, high frequency of mutation in melanoma and notable frequency of deep deletions in stomach adenocarcinoma; OR1J4-OR1J1, range of mutations, amplifications and deletions. OR, olfactory receptor. 


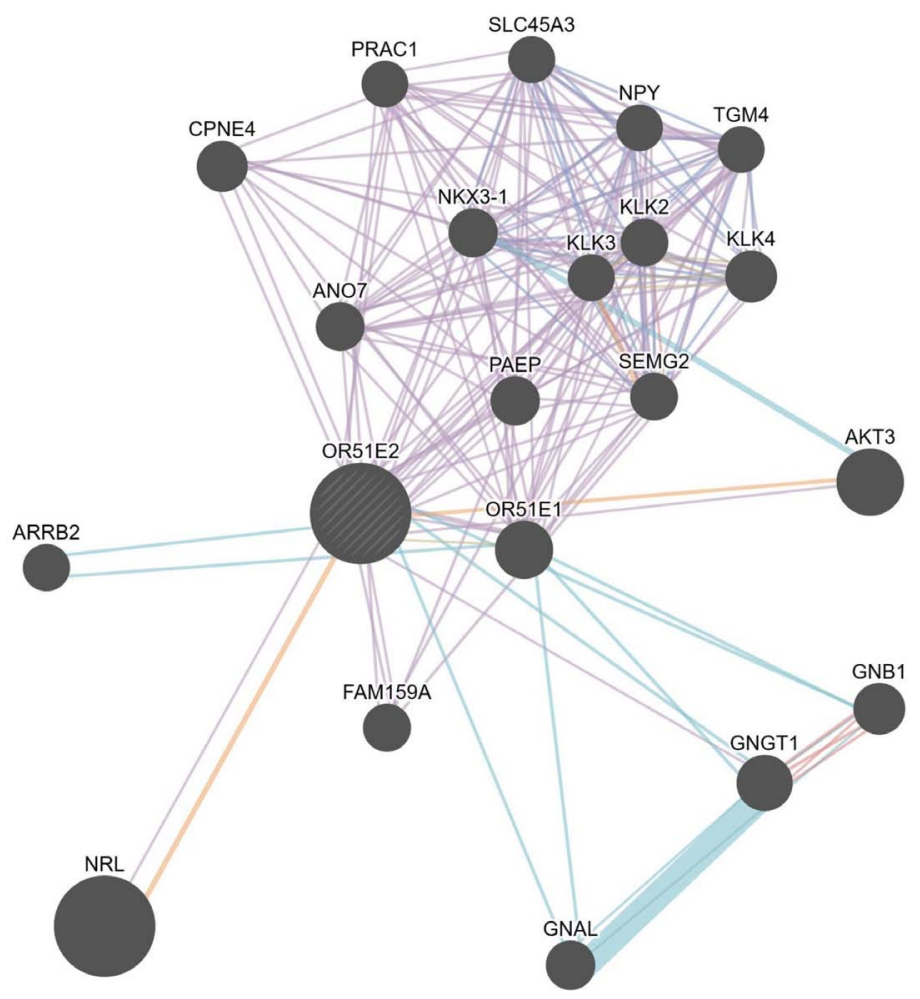

Figure 7. Network annotation of genes interacting with olfactory receptor OR51E2 (GeneMANIA). Visual representation of prostate specific genes and prostate specific biomarkers, such as KLK2 and KLK4, co-expressed (purple), have physical interactions (pink) and co-localised (blue) with OR51E2.

provided. Changes in DNA methylation can be one of the many steps towards malignant transformation (21). It is noteworthy that, among the studied ORs, OR51E2 was significantly overexpressed in PRAD with no apparent difference in the expression between Caucasian and African American groups. Notably, previous studies have implicated this OR in prostate cancer $(6,22)$, whilst 19 -hydroxyandrostenedione (a testosterone metabolite) is endogenously produced upon OR51E2 activation (23). Interestingly, it has been suggested that androgen sensitivity can be a determinant of the severity COVID-19 manifests (24). Moreover, increased TMPRSS2 expression has also been recorded for the prostate $(14,25)$. The noted high levels of TMPRSS 2 and CTSL expression, coupled with the presented data regarding OR expression (e.g., OR51E2) in malignant and healthy tissues suggest additional implications which merit further research; for example, regarding increased expression in the prostate and predisposition to severe COVID-19 documented in male patients $(14,25)$.

Interestingly, using the GeneMANIA software to gain better insight into the network of genes interacting with OR51E2, co-expression was noted with a plethora of genes related to prostate cancer (Fig. 7). One of the suggested interactions of this receptor is with KLK3 (kallikrein 3 or prostate specific antigen; PSA). KLK2 and KLK4 are also amongst the genes interacting with OR51E2 and are considered potential biomarkers for screening and monitoring of prostate cancer (26). Similarly, OR51E2 interactions were also noted with anoctamin 7 (ANO7), a prostate specific gene associated with aggressive disease; NKX3.1, a prostatic tumour suppressor gene; as well as prostate cancer susceptibility candidate 1 (PRAC1) $(27,28)$. Knockdown of
PRAC1 in human prostate epithelial stem cells has been shown to compromise their sphere formation in vitro (29). Furthermore, as a GPCR, OR51E2 interacts with arrestin $\beta 2$ (ARR $\beta 2$ ) which has been shown to be involved in $\beta-2$ adrenergic receptor signalling, inducing prostate cancer cell progression (30). Finally, OR51E2 also appears to interact with serine/threonine kinase 3 (AKT3), a well characterised kinase that has been shown to promote prostate cancer cell proliferation (31).

In conclusion, the present study offers new data regarding the expression of ORs in peripheral tissues and their co-expression pattern with key mediators of SARS-CoV-2 cell entry and infection (i.e., ACE2, TMPRSS2, and CTSL). Involvement of impaired OR signalling/function due to SARS-CoV-2 infection leading, not only in anosmia, but also in sequelae from other peripheral tissues (e.g., from the respiratory system or the prostate) is an intriguing hypothesis which merits further investigation in order to clarify the complete spectrum of OR functions that may be impaired in COVID-19. We acknowledge the limitations of our in silico approach, thus further in vitro, in vivo and clinical studies are clearly required to elucidate the role of ORs, both at the olfactory level and the periphery, in the context of COVID-19.

\section{Acknowledgements}

Not applicable.

\section{Funding}

No funding was received. 


\section{Availability of data and materials}

All data generated or analysed during this study are included in this published article (and its supplementary information files).

\section{Authors' contributions}

RK consulted the literature, analysed the data, produced the figures and contributed towards the manuscript. RK, $\mathrm{MH}, \mathrm{KC}$, HSR and DAS have contributed to the conception and design of the study, writing of the manuscript, providing revision and contributing towards final edits. IK and EK contributed equally to the conception of the work, data and literature analysis as well as interpretation. All authors read and approved the final manuscript.

\section{Ethics approval and consent to participate}

Not applicable.

\section{Patient consent for publication}

Not applicable.

\section{Competing interests}

DAS is the Editor-in-Chief for the journal, but had no personal involvement in the reviewing process, or any influence in terms of adjudicating on the final decision, for this article. The other authors declare that they have no competing interests.

\section{References}

1. World Health Organization (WHO): Coronavirus disease 2019 (COVID-19): situation report, 139. WHO, Geneva, 2020. https://www.who.int/emergencies/diseases/novel-coronavirus2019/situation-reports. Accessed June 8, 2020.

2. Pellegrino R, Cooper KW, Di Pizio A, Joseph PV, Bhutani S and Parma V: Corona Viruses and the Chemical Senses: Past, Present, and Future. Chem Senses bjaa031, 2020.

3. Mainland JD, Keller A, Li YR, Zhou T, Trimmer C, Snyder LL, Moberly AH, Adipietro KA, Liu WL, Zhuang H, et al: The missense of smell: Functional variability in the human odorant receptor repertoire. Nat Neurosci 17: 114-120, 2014.

4. Maßberg D and Hatt H: Human olfactory receptors: Novel cellular functions outside of the nose. Physiol Rev 98: 1739-1763, 2018.

5. An SS and Liggett SB: Taste and smell GPCRs in the lung: Evidence for a previously unrecognized widespread chemosensory system. Cell Signal 41: 82-88, 2018.

6. Neuhaus EM, Zhang W, Gelis L, Deng Y, Noldus J and Hatt H: Activation of an olfactory receptor inhibits proliferation of prostate cancer cells. J Biol Chem 284: 16218-16225, 2009.

7. Gosain R, Abdou Y, Singh A, Rana N, Puzanov I and Ernstoff MS: COVID-19 and cancer: a comprehensive review. Curr Oncol Rep 22, 53, 2020.

8. Hoffmann M, Kleine-Weber H, Schroeder S, Krüger N, Herrler T, Erichsen S, Schiergens TS, Herrler G, Wu NH, Nitsche A, et al: SARS-CoV-2 cell entry depends on ACE2 and TMPRSS2 and is blocked by a clinically proven protease inhibitor. Cell 181: 271-280.e8, 2020.

9. Walls AC, Park YJ, Tortorici MA, Wall A, McGuire AT and Veesler D: Structure, function, and antigenicity of the SARS-CoV-2 spike glycoprotein. Cell 181: 281-292.e6, 2020.

10. Yuki K, Fujiogi M and Koutsogiannaki S: COVID-19 pathophysiology: A review. Clin Immunol 215: 108427, 2020.

11. Butowt $\mathrm{R}$ and Bilinska K: SARS-CoV-2: olfaction, brain infection, and the urgent need for clinical samples allowing earlier virus detection. ACS Chem Neurosci 11: 1200-1203, 2020.
12. Brann DH, Tsukahara T, Weinreb C, Lipovsek M, Van den Berge $K$, Gong B, Chance R, Macaulay IC, Chou H, Fletcher R, et al. Non-neuronalexpression of SARS-CoV-2entry genesintheolfactory system suggests mechanisms underlying COVID-19-associated anosmia. bioRxiv: 10.1101/2020.03.25.009084

13. Chai P, Yu J, Ge S, Jia R and Fan X: Genetic alteration, RNA expression, and DNA methylation profiling of coronavirus disease 2019 (COVID-19) receptor ACE2 in malignancies: A pan-cancer analysis. J Hematol Oncol 13: 43, 2020.

14. Katopodis P, Anikin V, Randeva HS, Spandidos DA, Chatha K, Kyrou I and Karteris E: Pan-cancer analysis of transmembrane protease serine 2 and cathepsin $L$ that mediate cellular SARS-CoV-2 infection leading to COVID-19. Int J Oncol 57: 533-539, 2020.

15. Eliezer M, Hautefort C, Hamel AL, Verillaud B, Herman P, Houdart E and Eloit C: Sudden and complete olfactory loss function as a possible symptom of COVID-19. JAMA Otolaryngol Head Neck Surg: April 8, 2020 (Epub ahead of print).

16. Bilinska K, Jakubowska P, Von Bartheld CS and Butowt R: Von Bartheld CS and Butowt R: Expression of the SARS-CoV-2 entry proteins, ACE2 and TMPRSS2, in cells of the olfactory epithelium: identification of cell types and trends with age. ACS Chem Neurosci 11: 1555-1562, 2020.

17. Wang S, Zhou X, Zhang T and Wang Z: The need for urogenital tract monitoring in COVID-19. Nat Rev Urol 17: 314-315, 2020.

18. Reghunathan R, Jayapal M, Hsu LY, Chng HH, Tai D, Leung BP and Melendez AJ: Expression profile of immune response genes in patients with Severe Acute Respiratory Syndrome. BMC Immunol 6: 2, 2005.

19. Hu W, Yen YT, Singh S, Kao CL and Ba WH: SARS-CoV regulates immune function-related gene expression in human monocytic cells. Viral Immunol 25: 277-288, 2012.

20. VanLeuven JT, Ridenhour BJ, Gonzalez AJ, Miller CR and Miura TA: Lung epithelial cells have virus-specific and shared gene expression responses to infection by diverse respiratory viruses. PLoS One 12: e0178408, 2017.

21. Wajed SA, Laird PW and DeMeester TR: DNA methylation: An alternative pathway to cancer. Ann Surg 234: 10-20, 2001.

22. Xia C, Ma W, Wang F, Hua SB and Liu M: Identification of a prostate-specific G-protein coupled receptor in prostate cancer. Oncogene 20: 5903-5907, 2001.

23. Abaffy T, Bain JR, Muehlbauer MJ, Spasojevic I, Lodha S, Bruguera E, O'Neal SK, Kim SY and Matsunami H: A testosterone metabolite 19-hydroxyandrostenedione induces neuroendocrine trans-differentiation of prostate cancer cells via an ectopic olfactory receptor. Front Oncol 8: 162, 2018.

24. Wambier CG, Goren A, Vaño-Galván S, Ramos PM, Ossimetha A, Nau G, Herrera S and McCoy J: Androgen sensitivity gateway to COVID-19 disease severity. Drug Dev Res: May 15, 2020 (Epub ahead of print).

25. Stopsack KH, Mucci LA, Antonarakis ES, Nelson PS and Kantoff PW: TMPRSS2 and COVID-19: Serendipity or opportunity for intervention? Cancer Discov 10: 779-782, 2020.

26. Hong SK: Kallikreins as biomarkers for prostate cancer. BioMed Res Int 2014: 526341, 2014.

27. Kaikkonen E, Rantapero T, Zhang Q, Taimen P, Laitinen V, Kallajoki M, Jambulingam D, Ettala O, Knaapila J, Boström PJ, et al; PRACTICAL Consortium: ANO7 is associated with aggressive prostate cancer. Int J Cancer 143: 2479-2487, 2018.

28. Gurel B, Ali TZ, Montgomery EA, Begum S, Hicks J, Goggins M, Eberhart CG, Clark DP, Bieberich CJ, Epstein JI, et al: NKX3.1 as a marker of prostatic origin in metastatic tumors. Am J Surg Pathol 34: 1097-1105, 2010.

29. Hu WY, Hu DP, Xie L, Li Y, Majumdar S, Nonn L, Hu H, Shioda $\mathrm{T}$ and Prins GS: Isolation and functional interrogation of adult human prostate epithelial stem cells at single cell resolution. Stem Cell Res (Amst) 23: 1-12, 2017.

30. Zhang P, He X, Tan J, Zhou X and Zou L: $\beta$-arrestin 2 mediates $\beta-2$ adrenergic receptor signaling inducing prostate cancer cell progression. Oncol Rep 26: 1471-1477, 2011.

31. Lin HP, Lin CY, Huo C, Jan YJ, Tseng JC, Jiang SS, Kuo YY, Chen SC, Wang CT, Chan TM, et al: AKT3 promotes prostate cancer proliferation cells through regulation of Akt, B-Raf, and TSC1/TSC2. Oncotarget 6: 27097-27112, 2015.

This work is licensed under a Creative Commons Attribution-NonCommercial-NoDerivatives 4.0 International (CC BY-NC-ND 4.0) License. 